\title{
Nonlinear reflectivity of semiconductor microcavities in the weak- and strong-coupling regimes: Experiment and theory
}

\author{
A. L. Bradley, J. P. Doran, T. Aherne, and J. Hegarty \\ Physics Department, Trinity College, College Green, Dublin 2, Ireland \\ R. P. Stanley, R. Houdré, U. Oesterle, and M. Ilegems \\ Institüt de Micro- et Optoélectronique, EPFL, CH-1015 Lausanne, Switzerland
}

(Received 2 September 1997)

\begin{abstract}
We use an entirely classical model to describe the nonlinear properties of semiconductor microcavities in both the weak- and strong-coupling regimes, as measured in a series of spectrally and temporally resolved pump-probe experiments. The model, which follows on from a linear dispersion model previously used to describe the linear properties, provides very good qualitative agreement between a wide range of experimental and theoretical spectra. We also find that on the time scales of our observation (tens of picoseconds), the model provides an excellent description of the complex temporal evolution of the nonlinear spectra.
\end{abstract}

[S0163-1829(98)05816-0]

\section{INTRODUCTION}

The potential of semiconductor microcavities for physics, applications, and devices has already stimulated a great deal of research over the past five years. ${ }^{1,2}$ Central to the appeal of these wavelength scale cavity structures is the opportunity they provide for improved control of the light-matter interaction, in this case between optical photons and the semiconductor band-edge carriers or excitons. Two regimes of microcavity operation can be considered: the weak-coupling regime and the strong-coupling regime. In the more standard weak-coupling regime, the identity of the electronic states inside the cavity is not altered by the presence of the cavity and the photon-matter interaction can be described using a perturbative approach. In this weak-coupling regime, the presence of the microcavity can, however, have profound effects on the spatial and spectral characteristics of the spontaneous emission. ${ }^{3}$ Such effects have already been engineered to yield increases in the efficiency of planar lightemitting diodes. ${ }^{4}$

In the strong-coupling regime, on the other hand, the light-matter interaction inside the microcavity, in this case with the quantum-well exciton, is sufficiently strong that the exciton and photon states lose their individual identities and the normal modes of the coupled system become a pair of mixed exciton-photon states. These states, which have been labeled alternately as cavity polaritons ${ }^{5}$ or dressed excitons, ${ }^{6}$ have an energy splitting between them. The size of this energy splitting, or Rabi splitting $\left(\Omega_{R}\right)$, depends on the size of the exciton-photon interaction, which is characterized by the oscillator strength $(f)$ of the exciton transition. The splitting varies as $\Omega_{R} \propto \sqrt{f / L_{\text {cav }}}$. This phenomenon is analogous to vacuum Rabi splitting observed in atomic microcavity systems. ${ }^{7}$ In the case of strong coupling, a more dramatic alteration of the fundamental dynamical aspects of the excited states is possible than in the weak-coupling cavity. For example, the emission time of the cavity polariton is in principle the average of the exciton lifetime and the lifetime of the cavity photon. In the special circumstances of resonant pumping and very low excitation intensity, this alteration of the spontaneous emission lifetime from strong-coupling microcavities has been observed, ${ }^{8}$ although the situation is considerably more complex for the more general condition of nonresonant pumping. 9

Since the first observation in $1992,{ }^{10}$ a wide variety of linear optical measurements have been performed on strongcoupling microcavities aimed at attaining a correct description of the strongly coupled states. The simple quantummechanical mixed-oscillator picture that works well in the case of atomic microcavities is less satisfactory in the semiconductor case. Complications arise due to the "in-plane" part of the exciton wave function (which is not strongly coupled to the cavity photon $)^{11}$ and from the complicating effects of the inhomogeneous broadening which is difficult to avoid in quantum-well structures. ${ }^{12,13}$ In fact, this picture works best in the special case of resonant pumping, where only the small region of the exciton phase space that is strongly coupled to the photon is probed. Alternatively, many of the features in the linear optical response of strongcoupling microcavities, such as the presence of the Rabi splitting, can actually be understood within an entirely classical linear dispersion theory, as first demonstrated by Zhu et $a l .{ }^{14}$ for the case of atomic microcavity systems and then successfully applied to semiconductor microcavities by Houdré et al. $^{5}$ It is clear, as always, that the possible descriptions of the physical system depend on the measurement that is being interpreted.

More recently, nonlinear optical measurements have also been carried out and again a variety of physical pictures have been used to describe the nonlinear behavior of the strongly coupled system. Yamamoto et al. ${ }^{15}$ described the nonlinear behavior of the coupled system in terms of the transition from a dressed boson to a dressed fermion as the excitation density is increased. Hanamura identified the importance of the degree of inhomogeneous broadening to the nonlinear response and also described the system behavior with a quantum field theory approach. ${ }^{16}$ On the other hand, a 
much simpler classical description of the nonlinear behavior in terms of a straightforward saturation of the oscillator strength of the exciton was utilised by Houdre et al. ${ }^{17}$ and by Rhee et al. ${ }^{18}$ In this paper, we show that the classical treatment of Zhu et al. ${ }^{14}$ can be extended to describe consistently a detailed series of spectrally and temporally resolved nonlinear reflectivity measurements in semiconductor microcavities operating in both the weak- and strong-coupling regimes.

We present time-resolved pump-probe measurements performed in the reflection geometry on a pair of microcavity samples. The first sample contains a single quantum well and operates at the border of the weak- and strong-coupling regimes. The second sample contains six quantum wells and is clearly in the strong-coupling regime. Initially experimental results for the simpler, single-well, sample are presented. This is followed by a description of a model that we have developed to simulate the observed nonlinear spectra and their temporal evolution. We then present results on the strong-coupling sample and show that the same model can also be used to simulate this data. In all cases this classical model provides an excellent qualitative description of the data. Finally, we discuss why such a model works.

\section{SAMPLES AND EXPERIMENT}

Both microcavity samples are planar Fabry-Perot-type structures with multiple quarter-wave stack distributed Bragg reflectors (DBR's) in which the low-(high-) index material is AlAs $\left(\mathrm{Al}_{0.1} \mathrm{Ga}_{0.9} \mathrm{As}\right)$. The critical coupling microcavity, sample $A$, contains a single 75 - $\AA$-thick $\operatorname{In}_{0.13} \mathrm{Ga}_{0.87}$ As quantum well situated at the center of a $2 \lambda$ cavity, i.e., at the antinode of the electric-field standing wave. The front and back DBR's contain 7 and $19 \frac{1}{2}$ pairs respectively. The strong-coupling microcavity, sample B, has 6 and $19 \frac{1}{2}$ pairs in its front and back mirrors, respectively, and contains six $75-\AA \mathrm{In}_{0.13} \mathrm{Ga}_{0.87}$ As quantum wells in a $3 \lambda / 2$ cavity, three at each available electric-field antinode. A schematic illustration of sample B is shown in Fig. 1. As is normal with such microcavity samples, the wafers were not rotated during part of the molecular-beam-epitaxy growth of the cavity layer, leading to a wedge-shaped cavity which allows for detuning of the exciton and cavity resonances by moving across the sample. ${ }^{19}$ It is the presence of an anticrossing, rather than a crossing, of the cavity and exciton resonances at zero detuning that discriminates positively between strong- and weakcoupling samples.

The nonlinear pump-probe measurements were performed in the reflection geometry, as illustrated in Fig. 2(c). The excitation source was a Nd:YAG (yttrium aluminum garnet) pumped synchronously mode-locked Styryl-13 dye laser, which produced pulses of 20-70 ps in the $930 \mathrm{~nm}$ region. All of the measurements reported in this paper were carried out at the position of zero exciton-cavity detuning and at a sample temperature of $T=7 \mathrm{~K}$ in a closed-cycle helium cryostat. The nonlinear reflectivity spectra are differential spectra recorded using two cascaded lock-in amplifiers. The pump and probe beams are modulated at two different frequencies. The first lock-in amplifier detects the probe and the second detects the modulation due to the pump.

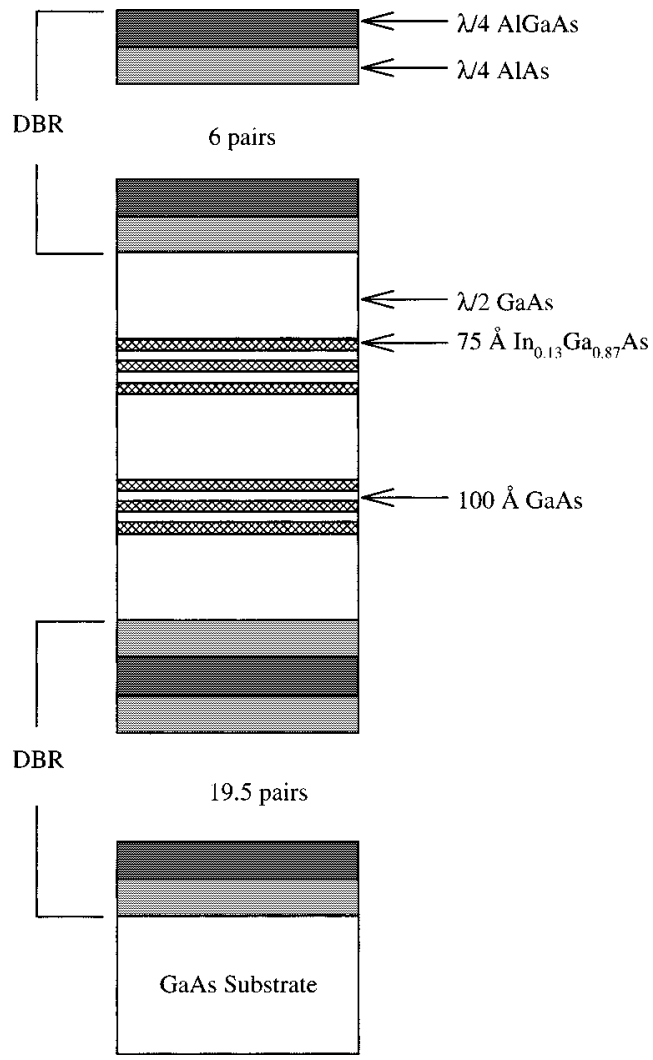

FIG. 1. Schematic showing structure of strong-coupling microcavity (sample B).

\section{RESULTS}

\section{Single-well sample}

The linear reflectivity of sample A, as measured at the position of zero detuning between the exciton and photon, exhibits the single resonance expected for a weak or critical coupling sample and is shown in Fig. 2(a). This spectrum is recorded using the probe beam, the intensity of which is selected to be low enough to exclude nonlinearities. Figure 2(b) shows the nonlinear reflectivity spectra recorded, for a range of pump beam intensities, at zero delay between the pump and probe pulses. A positive signal corresponds to an increase of the reflectivity over its low intensity (linear) value and a negative signal to a reduction in the reflectivity. It is clear that for this sample there is a large intensity dependence on the general shape of the nonlinear spectra, as well as in the actual size of the signal. At the lowest pump intensities the spectrum is almost symmetric about the resonance, but becomes increasingly asymmetric as the intensity is raised.

As indicated in the Introduction, we will use a classical linear dispersion theory to describe our results. In this approach, the microcavity structures (DBR's and cavity) are modeled in the standard manner by solution of Maxwell's equations, with appropriate use of boundary conditions at each interface. Our method allows the straightforward calculation of the electric-field intensity at all positions inside the structures, as well as the absorption, reflectivity, and transmission spectra of the structure. The quantum-well exciton can be described in the simplest approximation as a classical Lorentz oscillator with a dispersive dielectric constant $\varepsilon$ : 


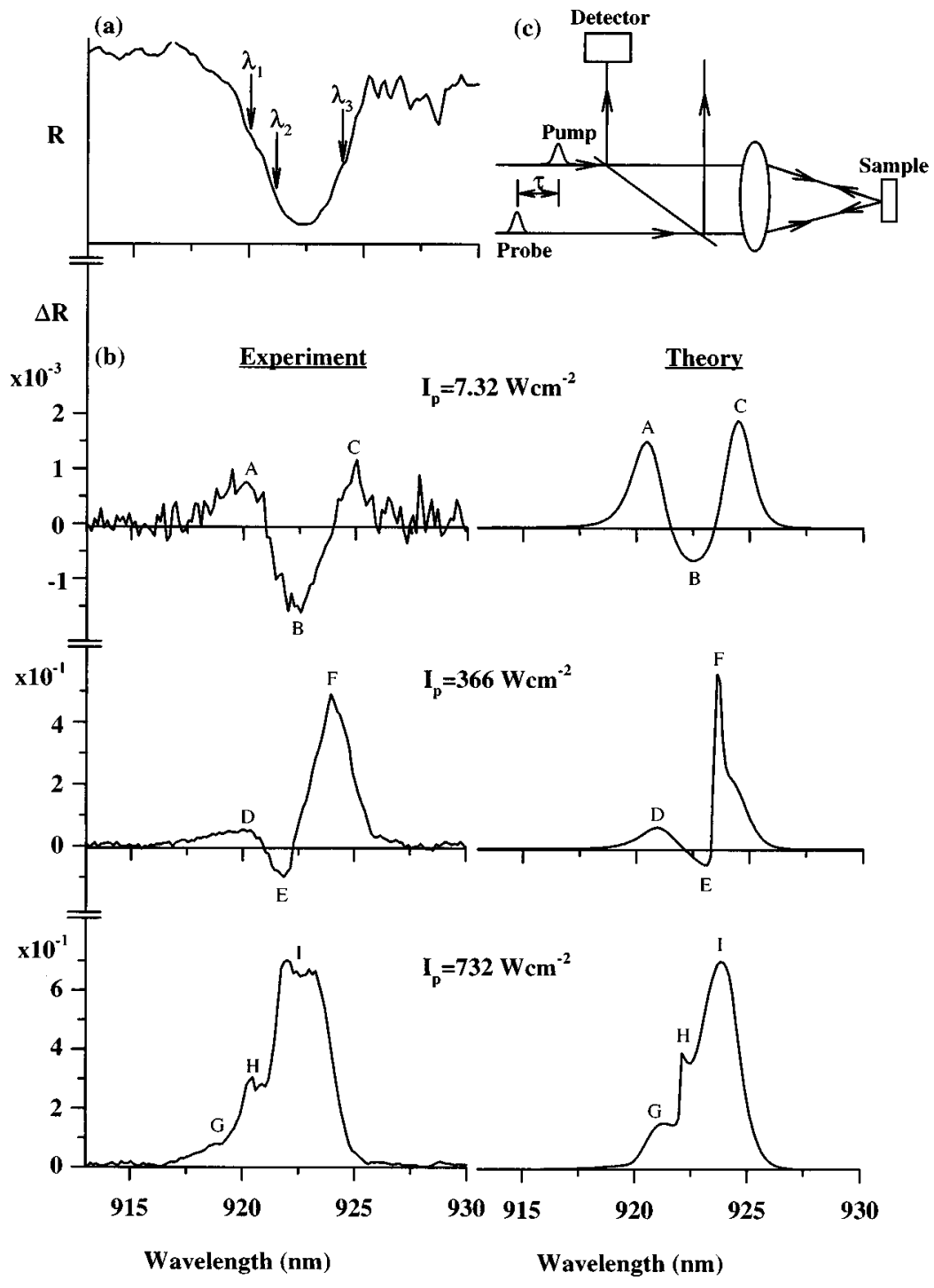

FIG. 2. (a) Linear reflectivity spectrum of sample A. (b) Experimental (left-hand side) and theoretical (right-hand side) nonlinear reflectivity spectra of sample A at varying pump intensities $(\tau=0)$. (c) Configuration of the pump-probe experiment.

$$
\varepsilon(E)=\varepsilon_{\infty}+\frac{f q^{2} \mathrm{~h}^{2}}{m \varepsilon_{0} L_{z}} \frac{1}{E_{X}^{2}-E^{2}-i \gamma E},
$$

where $E$ is the energy, $f$ is the oscillator strength per $\mathrm{cm}^{2}, q$ is the charge, $m$ the mass, $E_{X}$ the energy of the exciton resonance, $\gamma$ the homogeneous exciton linewidth, and $L_{z}$ the quantum-well thickness. The dielectric constant yields the refractive index of the quantum-well layer, the imaginary part of which gives the exciton absorption. At low intensities the exciton is described by Eq. (1), with the linear exciton parameters. These parameters are obtained by reproduction of the linear reflection spectrum of sample A [Fig. 2(a)]. Inhomogeneous broadening which results from alloy broadening and quantum-well thickness fluctuations must also be included in the calculation. We assume this broadening to be Gaussian. The linear exciton parameters we find are $E_{X 0}$ $=1.344 \mathrm{eV}, f_{0}=8.4 \times 10^{12} \mathrm{~cm}^{-2}$, and a homogeneous exciton linewidth $\gamma_{0}=0.05 \mathrm{meV}$; an inhomogeneous broadening of $3 \mathrm{meV}$ is also included. All of these parameters correspond well with values measured previously on these samples. ${ }^{5}$

The nonlinear properties are modeled using an extension of this approach. The presence of the pump beam, of intensity $I_{\mathrm{inc}}$, on the sample can alter the description of the exci- ton in Eq. (1) by modifying any of the exciton oscillator parameters: oscillator strength $f_{0}$, energy $E_{X 0}$, or linewidth $\gamma_{0}$. The combination of the cavity and the "new" exciton produces a "new" reflectivity spectrum, and the difference between this spectrum and the original linear spectrum corresponds to the differential reflectivity spectrum that we measure in the pump-probe experiment. It remains to determine how the exciton parameters vary with incident intensity.

The nonlinear behavior of excitons in quantum wells has been extensively investigated and the dominant nonlinear mechanisms, such as the phase-space filling, screened exchange interaction, and exciton scattering, which lead to saturation of the exciton oscillator strength, shifting of the exciton energy, and line broadening, are very well known. ${ }^{20}$ Our approach is to impose these well understood nonlinearities on the quantum-well exciton and proceed in the manner outlined above.

We consider two features in the nonlinear response as the most important. The first process is the saturation of the exciton oscillator strength as the excitation intensity at the quantum well $I_{\mathrm{qw}}$ increases. This saturation of the oscillator strength is due to both phase-space filling and screened exchange effects, and is described by ${ }^{20,21}$ 


$$
f=\frac{1}{1+I_{\mathrm{qw}} / I_{S}},
$$

where $I_{S}$ is a saturation intensity which corresponds to some saturation exciton density $N_{S}$.

The second important feature is a small blueshift of the exciton which comes about due to the screened exchange interaction. ${ }^{22}$ In bulk materials, this blueshift is canceled by a redshift arising due to long-range Coulomb interaction, but in quantum wells the latter contribution is less significant, resulting in a net blueshift which is a function of the exciton density. It is not known what precise dependence of the exciton blueshift on the pump intensity is expected, but in this work we find best agreement when we include an exciton blueshift $\delta E_{X}$ following a greater than linear dependence on the incident intensity. We incorporate a quadratic dependence

$$
\delta E_{X}=b I_{\mathrm{qw}}^{2},
$$

where $b$ is a constant. At very high excitation densities, the exciton blueshift will become comparable to the exciton binding energy, in which case the exciton dissociates. The above equation is therefore only meaningful for exciton blueshifts which are less than the binding energy.

A correct description of our experimental data also requires careful incorporation of the dynamical evolution of the reflectivity over the excitation pulse. Consider an instant along the pump pulse: there is a certain light intensity incident $I_{\text {inc }}$ on the sample, but the action of the cavity leads to quite a different light intensity inside the cavity at the position of the quantum well, $I_{\mathrm{qw}}$. Furthermore, the intensity at the quantum-well position is highly wavelength dependent. The modification of the exciton oscillator parameters leads to a change in the reflectivity spectrum of the structure for the light that arrives at the next instant of the pulse. Hence the distribution of the light field inside the cavity changes-it evolves over the duration of the pulse. These temporal effects are important, since the time scale of the pulse (tens of picoseconds) is much longer than the natural timescale of the cavity ( $\approx 1 \mathrm{ps})$.

In summary, the flow of the calculation is as follows: the initial parameters of the model are chosen in a manner that reproduces the linear reflectivity spectrum; then, progressing along the pulse in 1-ps steps, at each wavelength the light intensity at the quantum well is calculated; using Eqs. (2) new exciton oscillator parameters are found; these parameters are fed back into the model, thus allowing a calculation of the light intensity at the quantum well at the next instant of time. Following this iterative process to the peak of the excitation pulse, a reflectivity spectrum is produced, which then allows calculation of a nonlinear spectrum by subtraction of the linear spectrum. The step size of $1 \mathrm{ps}$ is chosen, as it corresponds to that step size below which smaller step sizes yield identical results. It is emphasized that this procedure is essential in order to reproduce all the intensitydependent features of the experimental data in both of our samples in a consistent manner.

The model is initially used to simulate experimental results for sample A, and the results are shown on the righthand side of Fig. 2(b). In the case of the lowest pump intensity $I_{\text {inc }}=7.32 \mathrm{~W} \mathrm{~cm}^{-2}$, the blueshift of the exciton is very small and the saturation of the oscillator strength is the dominant effect. The saturation density we have used is $N_{S}=2.9$ $\times 10^{10} \mathrm{~cm}^{-2}$, which corresponds closely to the value found by Houdré et al. ${ }^{17}$ A constant of $8.4 \times 10^{-26} \mathrm{~nm} /\left(\mathrm{cm}^{-2}\right)^{2}$, also given in terms of the density, was used in the expression for calculating the blueshift of the exciton. As can be seen in the figure, each of the features (labeled $A, B$, and $C$ ) in the experimental spectra are reproduced in the theoretical spectrum. At the higher pump intensities of $I_{\mathrm{inc}}=3.66$ $\times 10^{2} \mathrm{~W} \mathrm{~cm}^{-2}$ and $I_{\text {inc }}=7.32 \times 10^{2} \mathrm{~W} \mathrm{~cm}^{-2}$, the asymmetry in the spectrum comes from the increasing importance of the exciton blueshift at these intensities. Again, all of the features of the experimental data $(D-I)$ are reproduced in the calculation. In reproducing this intensity dependence of the shape and size of the nonlinear spectra, the values of $N_{S}$ and $b$ in Eqs. (2) are fixed and the incident intensity is varied according to the experimental conditions. The qualitative agreement is excellent.

\section{Time-resolved pump probe}

Time-resolved pump-probe experiments have also been performed and the temporal evolution of the nonlinear reflectivity exhibits a complex behavior that depends critically on the wavelength at which the measurement is carried out. Figure 3 shows the time-resolved experimental data as recorded at three different wavelengths [indicated by the arrows in Fig. 2(a)] across the resonance on sample A, for a pump intensity of $I_{\text {inc }}=3.66 \times 10^{2} \mathrm{~W} \mathrm{~cm}^{-2}$. At $\lambda_{1}$, which is on the high-energy side of the resonance, the signal is zero at zero delay, then decays rapidly to a negative value, and then recovers much more slowly. At $\lambda_{2}$ the signal is positive at zero delay, followed by a rapid decay from a positive to a negative value, and then once again recovering more slowly to zero. The final example shown (at $\lambda_{3}$ ) is toward the lowenergy side of the resonance and is a more straightforward decay (although highly nonexponential) of a positive signal to zero.

In order to adapt our classical model to reproduce these time-resolved data, the simplest assumption that can be made is that the nonlinear behavior will be governed simply by the decay of the excited population. In other words, the saturation of the exciton oscillator strength and the blueshift of the exciton energy due to the excitation density created by the optical pulse, as determined by Eqs. (2), will both recover to their linear values as this excitation density decays. Hence the reflectivity spectrum will recover its linear form. We have independently measured the decay of the excitation density using time-resolved photoluminescence. ${ }^{23}$ Under the same conditions of excitation density and temperature, the decay of the excited population of sample $\mathrm{A}$ is found to be single exponential with a time constant of $450 \mathrm{ps}$. Using this single exponential in conjunction with Eq. (2), the temporal evolution of $f$ and $E_{X}$ is ascertained, and hence that of the sample reflectivity. The theoretical spectra obtained using this approach are shown on the right-hand side of Fig. 3 and it can be seen that extremely good correspondence with the experimental data is found for each of the wavelengths. The precise shape of the evolution of each of these spectra depends on the detailed interplay of the exciton and cavity system, but come very straightforwardly from our model. 


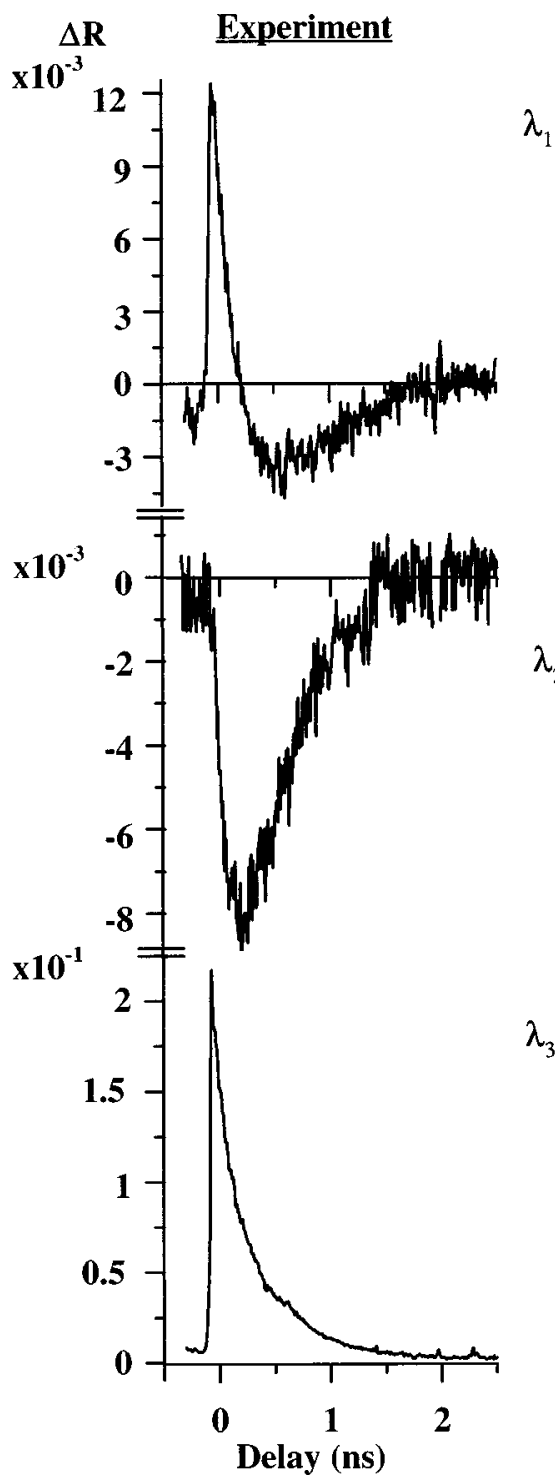

Six-well sample

The above sections have shown that the nonlinear properties of our single-well sample, which operates in the critical coupling regime, are very well explained with a relatively simple classical model. We now turn to sample $B$, the strong-coupling sample, which contains six quantum wells. Figure 4(a) shows the linear reflectivity spectrum of this sample recorded at the position of zero exciton-cavity detuning. The Rabi-split doublet identifying strong coupling is clearly seen. Nonlinear reflectivity spectra recorded for zero delay between the pump and probe pulses are shown in Fig. 4(b) for three different pump intensities. In this sample, while there is a change in the size of the nonlinear signal with pump intensity, the intensity dependence of the shape of the spectrum is minor.

We apply the same classical model to this sample, using the same exciton parameters as were used for the single-well sample. The calculated linear reflectivity spectrum in this sample is shown in Fig. 4(c) and, although not identical, is quite similar to the experimental spectrum, reproducing the Rabi doublet. Calculated nonlinear reflectivity spectra are shown on the right-hand side of Fig. 4(b). It should be emphasized that, in going from calculations on the single-well
FIG. 3. Experimental and theoretical evolution of the nonlinear reflectivity of sample A at three different pump wavelengths [as indicated in Fig. 2(a)]. sample to the six-well sample, only the sample structure is changed and all of the exciton oscillator parameters and the nonlinear parameters $\left[I_{\text {sat }}\right.$ and $b$ in Eqs. (2) $]$ are the same. Within this model there is no real distinction between the different regimes of microcavity operation-in all cases the microcavity is viewed as an oscillator within a Fabry-Perottype filter.

The theoretical spectra in Fig. 4(b) show good qualitative agreement with the experimental data, in particular the midintensity and high-intensity spectra. Each experimental spectrum contains several positive and negative features, labelled $A-L$ in Fig. 4(b). In all cases there is a large positive feature in the region of the lower-energy resonance and a series of weaker features in the region of the higher-energy resonance. If, for example, the spectrum corresponding to a pump intensity of $I_{\mathrm{inc}}=366 \mathrm{~W} \mathrm{~cm}^{-2}$ is examined, it can be seen that there is a remarkable correspondence between the existence of these features in the experimental and theoretical spectra. These features in the spectra come about due to the subtle interplay of the exciton and cavity and the inclusion of the dynamical evolution of the system over the excitation pulse is essential in reproducing the results. Quantitative agreement of these spectra with the experiment is not as good for 
(a)

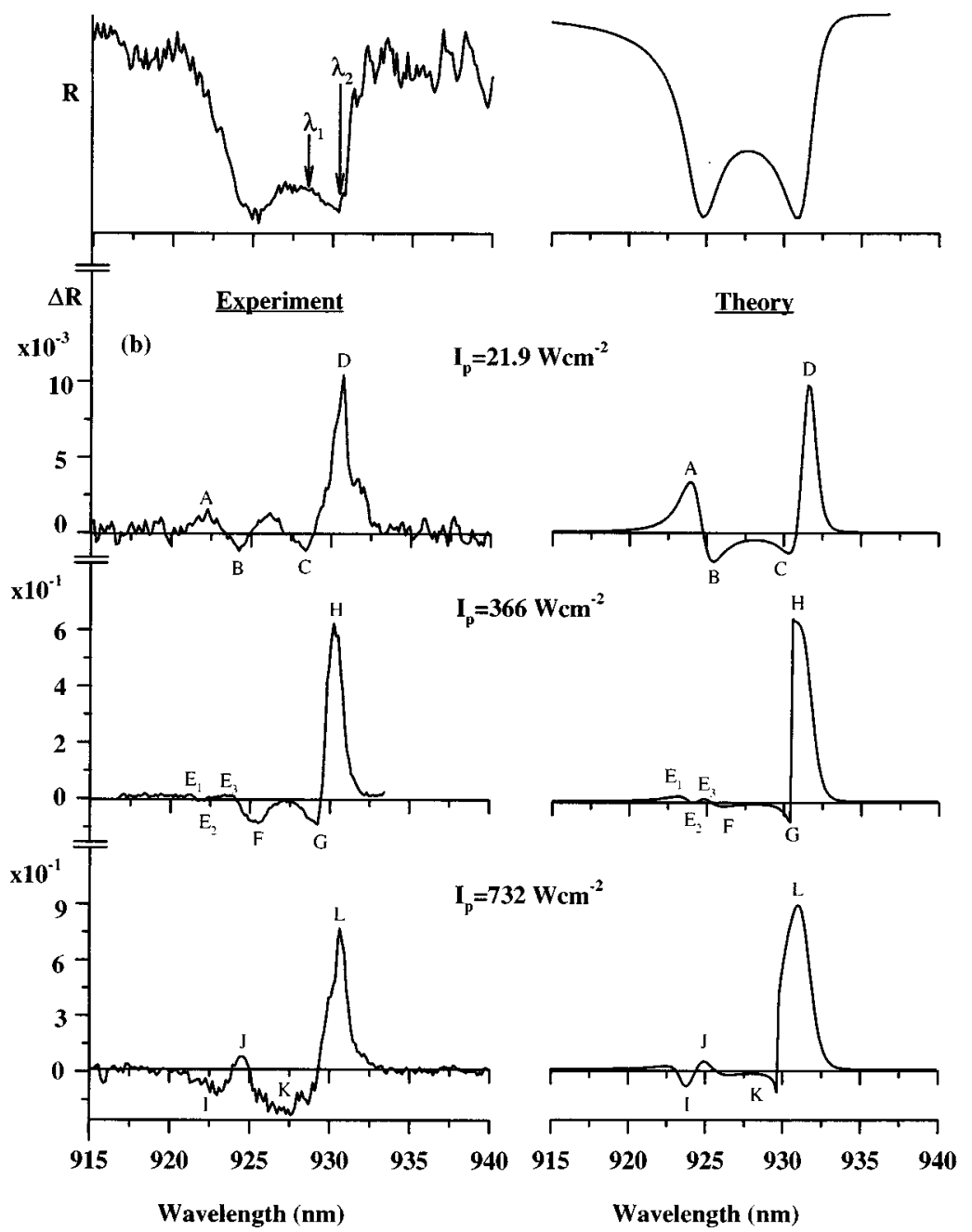

FIG. 4. (a) Linear reflectivity spectrum of sample B. (b) Experimental (left-hand side) and theoretical (right-hand side) nonlinear reflectivity spectra of sample B at varying pump intensities $(\tau=0)$. (c) Theoretical linear reflectivity spectra of sample B. sample B, as it was for sample A, and the lack of precise reproduction of the linear reflectivity spectrum may underlie this discrepancy. Nevertheless, the qualitative agreement is impressive.

Figure 5 shows time-resolved pump-probe data recorded on sample B at two different wavelengths [indicated by the arrows in Fig. 4(a)]. The same procedure as before is used for modeling the temporal evolution of the spectra in the strong-coupling sample. In this case the excited population is found, using time-resolved photoluminescence, to exhibit a single exponential decay with a decay time of $900 \mathrm{ps}^{23} \mathrm{In}-$ corporation of this into the model produced the data on the right-hand side of Fig. 5. Again, the agreement with experiment is excellent.

\section{DISCUSSION}

It is clear for sample $\mathrm{A}$, in the weak-coupling regime, we that expect linear dispersion theory to work. The nonlinear aspects are associated with the saturation and blueshift of the exciton, while the effect of the cavity is a linear filtering of the pump and probe pulses both spectrally and temporally. Although the measured nonlinear spectra show a wealth of features, the underlying physics is unchanged with respect to that of bare quantum-well excitons.

While one might expect that this should no longer be the case for sample B, in the strong-coupling regime, as we have shown, the same model works without recourse to more sophisticated or exotic modeling. This is not entirely unsurprising for the following reasons.

First, the linear dispersion theory is a very good model. All the qualities of a classical two-level system are embedded in the macroscopic refractive index (dielectric constant) given in Eq. (1). As the classical and quantum-mechanical treatments of two-level systems give identical results, we can then expect the linear dispersion theory to work equally well in both strong- and weak-coupling regimes. Of course, when the effect of in-plane dispersion becomes important, then the simple two-level model (in either its quantum or classical guise) is no longer appropriate.

Second, we found no new nonlinear mechanisms associated with the strong coupling to be apparent in this type of pump-probe experiment. Therefore, if the linear dispersion theory gives an accurate description of the linear spectra in the strong-coupling regime, introducing the excitonic nonlinearities into the model will describe the nonlinear behavior.

Finally, the time scale on which strong-coupling effects are important (i.e., the shorter of the photon lifetime and the exciton dephasing time) are much shorter than the pulse lengths used. This experiment well may be blind to the more exotic phenomena that have been proposed by Norris ${ }^{24}$ and Yammamoto. ${ }^{15}$ 


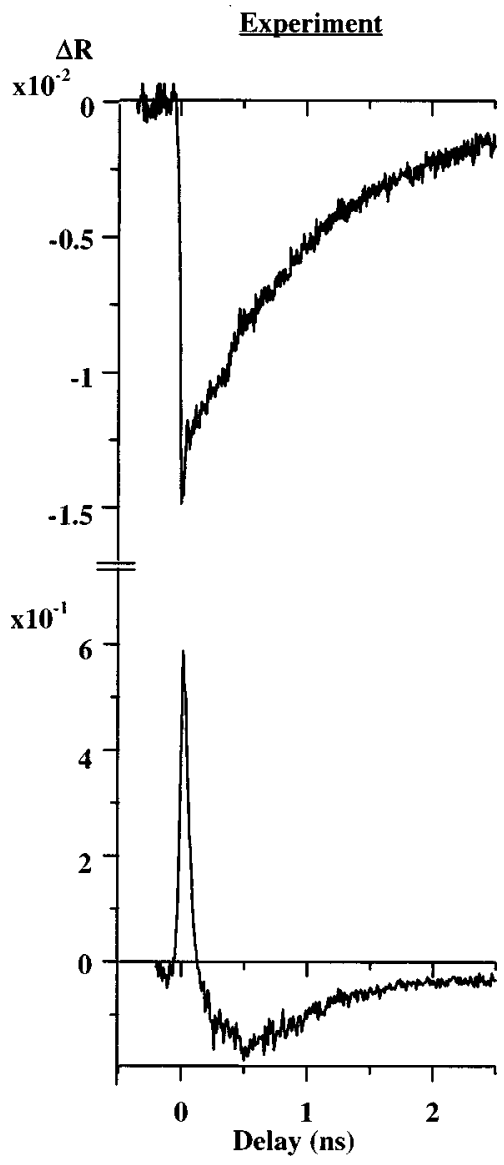

\section{CONCLUSIONS}

We have measured the nonlinear reflectivity of semiconductor microcavities, operating both in the critical coupling and in the strong-coupling regimes, using a time-resolved pump-probe technique and have approached the description of our results using a classical model which is an extension of the linear dispersion model previously used to predict linear optical properties. The qualitative agreement of this model with experiment is very good, reproducing all of the main spectral and temporal features of the data in a consistent manner over a range of pump intensities in both samples.

These results show that the classical treatment of the strong-coupling regime in describing the nonlinear properties
FIG. 5. Experimental and theoretical evolution of the nonlinear reflectivity of sample B at two different pump wavelengths [as indicated in Fig. 4(a)].
${ }^{1}$ Confined Electrons and Photons, edited by E. Burstein and C.

Weisbuch (Plenum, New York, 1995).

${ }^{2}$ Microcavities and Photonic Bandgaps: Physics and Applications, edited by J. Rarity and C. Weisbuch (Kluwer, Dordrecht, 1996).

${ }^{3}$ G. Bjork, S. Machida, Y. Yamamoto, and K. Igeta, Phys. Rev. A 44, 669 (1991).

${ }^{4}$ H. deNeve, J. Blondelle, P. Van Daele, G. Borghs, P. Demeester, and R. Baets, Appl. Phys. Lett. 70, 7 (1997); J. Blondelle, H. deNeve, P. Demeester, P. Van Daele, G. Borghs, and R. Baets, Electron. Lett. 31, 1286 (1995).

${ }^{5}$ R. Houdré, R. P. Stanley, U. Oesterle, M. Ilegems, and C. Weisbuch, Phys. Rev. B 49, 16761 (1994). is sufficient on the time scales with which we deal in our experiments, i.e., tens of picoseconds. Under these conditions the strong-coupling microcavity corresponds to an asymmetric Fabry-Perot modulator, ${ }^{25}$ which contains a strong oscillator and operates beyond the point of impedance matching. It is emphasized, however, that this model does not address issues of dynamics. We were able to describe all of the observed nonlinear dynamics in terms of the relaxation of the excited population, which was measured independently and which occurred on a long time scale (hundreds of picoseconds). Alterations in the fundamental excited-state dynamics that have been observed under conditions of resonant pumping and very low excitation intensities require a more elaborate description of the strong-coupling regime.
${ }^{6}$ S. Pau, G. Bjork, J. Jacobson, H. Cao, and Y. Yamamoto, Phys. Rev. B 51, 7090 (1995).

${ }^{7}$ Cavity Quantum Electrodynamics, edited by P. R. Berman (Academic, Boston, 1994).

${ }^{8}$ B. Sermage, S. Long, I. Abram, J. Y. Marzin, J. Bloch, R. Planel, and V. Thierry-Mieg, Phys. Rev. B 53, 16516 (1996).

${ }^{9}$ J. P. Doran, A. L. Bradley, B. Roycroft, T. Aherne, J. Hegarty, R. P. Stanley, R. Houdre, U. Oesterle, and M. Ilegems, Microcavities and Photonic Bandgaps: Physics and Applications (Kluwer, Dordrecht, 1996), p. 59.

${ }^{10}$ C. Weisbuch, M. Nishioka, A. Ishikawa, and Y. Arakawa, Phys. Rev. Lett. 69, 3314 (1992). 
${ }^{11}$ R. Houdre, C. Weisbuch, R. P. Stanley, U. Oesterle, P. Pellandini, and M. Ilegems, Phys. Rev. Lett. 73, 2043 (1994).

${ }^{12}$ R. Houdre, R. P. Stanley, and M. Ilegems, Phys. Rev. A 53, 2711 (1996)

${ }^{13}$ J. Jacobson, S. Pau, H. Cao, G. Bjork, and Y. Yamamoto, Phys. Rev. A 51, 14437 (1995).

${ }^{14}$ Y. Zhu, D. J. Gauthier, S. E. Morin, Q. Wu, H. J. Carmichael, and T. W. Mossberg, Phys. Rev. Lett. 64, 2499 (1990).

${ }^{15}$ Y. Yamamoto, J. Jacobson, S. Pau, H. Cao, and G. Bjork, in Microcavities and Photonic Bandgaps: Physics and Applications (Ref. 2), p. 457.

${ }^{16}$ E. Hanamura, in Microcavities and Photonic Bandgaps: Physics and Applications (Ref. 2), p. 533.

${ }^{17}$ R. Houdre, J. L. Gibernon, P. Pellandini, R. P. Stanley, U. Oesterle, C. Weisbuch, J. O'Gorman, and M. Ilegems, Phys. Rev. B 52, 7810 (1995).

${ }^{18}$ J. K. Rhee, R. Lai, T. B. Norris, Y. Arakawa, and M. Nishioka, Proc. Quantum Electron. 60 (1995).

${ }^{19}$ R. P. Stanley, R. Houdre, C. Weisbuch, U. Oesterle, and M. Ilegems, Phys. Rev. B 53, 10995 (1996).
${ }^{20}$ S. Schmitt-Rink, D. S. Chemla, and D. A. B. Miller, Adv. Phys. 38, 89 (1989).

${ }^{21}$ A. Miller, D. A. B. Miller, and S. D. Smith, Adv. Phys. 30, 697 (1981).

${ }^{22}$ N. Peyghambarian, H. M. Gibbs, J. L. Jewell, A. Antonetti, A. Migus, D. Hulin, and M. Mysyrowicz, Phys. Rev. Lett. 53, 2433 (1984).

${ }^{23}$ The two samples have different decay times as measured using time-resolved photoluminescence. There are many factors which influence the decay of the excited population. A comprehensive study of time-resolved photoluminescence has been undertaken and complex behavior has been observed. The results of these investigations are soon to be published by B. Roycroft, J. P. Doran, J. Hegarty, R. P. Stanley, R. Houdre, U. Oesterle, and M. Ilegems.

${ }^{24}$ T. B. Norris, J. K. Rhee, R. Lai, D. S. Citrin, M. Nishioka, and Y. Arakawa, Prog. Cryst. Growth Charact. Mater. 33, 155 (1996).

${ }^{25}$ K. K. Law, M. Whitehead, J. L. Mertz, and L. A. Coldren, Electron. Lett. 27, 1683 (1991). 